\title{
Goodness-of-fit test for the logistic distribution based on multiply type-II censored samples
}

\author{
Suk-Bok Kang ${ }^{1} \cdot$ Jun-Tae Han $^{2} \cdot$ Young-Seuk Cho ${ }^{3}$ \\ ${ }^{1}$ Department of Statistics, Yeungnam University \\ ${ }^{2}$ Research Management Team, Korea Student Aid Foundation \\ ${ }^{3}$ Department of Statistics, Pusan National University \\ Received 20 November 2013, revised 3 December 2013, accepted 9 December 2013
}

\begin{abstract}
In this paper, we derive the estimators of the location parameter and the scale parameter in a logistic distribution based on multiply type-II censored samples by the approximate maximum likelihood estimation method. We use four modified empirical distribution function (EDF) types test for the logistic distribution based on multiply type-II censored samples using proposed approximate maximum likelihood estimators. We also propose the modified normalized sample Lorenz curve plot for the logistic distribution based on multiply type-II censored samples. For each test, Monte Carlo techniques are used to generate the critical values. The powers of these tests are also investigated under several alternative distributions.
\end{abstract}

Keywords: Approximate maximum likelihood estimator, goodness-of-fit test, logistic distribution, modified normalized sample Lorenz curve, multiply type-II censored sample.

\section{Introduction}

The probability density function (pdf) and the cumulative distribution function (cdf) of the logistic distribution are given by

$$
f(x ; \sigma, \theta)=\frac{\pi}{\sigma \sqrt{3}} \frac{\exp \left(-\frac{\pi(x-\theta)}{\sigma \sqrt{3}}\right)}{\left[1+\exp \left(-\frac{\pi(x-\theta)}{\sigma \sqrt{3}}\right)\right]^{2}},-\infty<x<\infty, \sigma>0,
$$

and

$$
F(x ; \sigma, \theta)=\frac{1}{1+\exp \left(-\frac{\pi(x-\theta)}{\sigma \sqrt{3}}\right)}, \quad-\infty<x<\infty,
$$

where $\sigma$ and $\theta$ are scale and location parameters respectively.

\footnotetext{
${ }^{1}$ Professor, Department of Statistics, Yeungnam University, Gyeongsan 712-749, Korea

2 Corresponding author: Manager, Research Management Team, Korea Student Aid Foundation, Seoul 100-753, Korea. E-mail: hanjt@kosaf.go.kr

${ }^{3}$ Professor, Department of Statistics, Pusan National University, Busan 609-735, Korea
} 
The logistic distribution has been one of the most important statistical distributions because of its simplicity. It has been used for growth models and as a substitute for the normal distribution.

Order statistics from the logistic distribution and their moments were first examined by Birnbaum and Dudman (1963). Mathai (2003) studied some general moments of order statistic in the logistic distribution. Mathai (2003) also evaluated survival function and reliability in the logistic distribution.

The most common censoring schemes are type-I and type-II censoring, but the conventional type-I and type-II censoring schemes do not have flexibility. Multiply type-II censoring is a generalization of type-II censoring. Multiply type-II censored sampling arises in a lifetesting experiment whenever the experimenter does not observe the failure times of some units placed on a like-test. Another situation where multiply censored samples arise naturally is when some units failed between two points of observation with the exact times of failure of these units unobserved.

The approximate maximum likelihood estimating method was first developed by Balakrishnan (1989) for the purpose of providing explicit estimators of the scale parameter in the Rayleigh distribution. It has been noted that in most cases, the maximum likelihood method does not provide explicit estimators based on censored samples. When the sample is multiply censored, the maximum likelihood method does not admit explicit solutions. Therefore, it is desirable to develop which approximations to this maximum likelihood method would provide us with estimators that are explicit functions of order statistics. Balakrishnan et al. (1995) obtained the best linear unbiased estimators (BLUEs), the maximum likelihood estimators and the approximate maximum likelihood estimators (AMLEs) in a logistic distribution based on multiply type-II censored samples. Fei et al. (1995) studied the estimation for the two-parameter Weibull distribution and extreme-value distribution under multiply type-II censoring. They compared the mean squared errors of the maximum likelihood estimators, AMLEs, and BLUEs of the parameters in the extreme value distribution. Maswadah (2003) derived the conditional confidence intervals for the parameters based on the generalized order statistics. Balakrishnan et al. (2004) discussed point and interval estimation for the extreme value distribution under progressively type-II censoring. Balakrishnan and Kateri (2008) proposed a simple graphical solution for the determination of the maximum likelihood estimator of the shape parameter in the Weibull distribution. Kang et al. (2008) derived the AMLEs of the scale parameter in the half-logistic distribution under progressive type-II censoring. They also proposed the estimators of the reliability function by using the proposed estimators of the parameters. Han and Kang (2008) derived the AMLEs of the scale parameter and the location parameter in a double Rayleigh distribution based on multiply type-II censored samples. Recently, Shin and Lee (2012) suggested an estimation method of the parameter in an exponential distribution based on a progressive type-I interval censored sample with semi-missing observation. Kim et al. (2011a, 2011b) suggested Bayesian estimations under type-I hybrid and progressively type-II censoring.

Porter III et al. (1992) developed three modified Kolmogorov-Smirnov, Anderson-Darling, and Cramer-von Mises tests for the Pareto distribution with unknown parameters of the location and scale and known shape parameter based on the complete samples. Puig and Stephens (2000) studied some tests of fit for the Laplace distribution based on the empirical distribution function (EDF) statistics and the application of the Laplace distribution in the least absolute deviations regression. Choulakian and Stephens (2001) studied the estimation 
of parameters and goodness-of-fit tests for the generalized Pareto distribution.

In this paper, we derive the AMLEs of the location parameter $\theta$ and the scale parameter $\sigma$ in a logistic distribution under multiply type-II censored sample. We use four modified EDF type tests, including the modified Kolmogorov-Smirnov test, the modified AndersonDarling test, the modified Cramer-von Mises test, and the modified Watson test for the logistic distribution with unknown parameters based on multiply type-II censored samples using the proposed estimators and EDF. We propose the modified normalized sample Lorenz curve (NSLC) plot to test for the logistic distribution based on multiply type-II censored samples.

For each test, Monte Carlo techniques are used to generate the critical values. The powers of these tests are also investigated under lognormal, exponential, Rayleigh, double Rayleigh alternative distributions.

\section{Approximate maximum likelihood estimators}

We assume that $n$ items are put on a life test, but only $a_{1}$ th, $a_{2}$ th, $\ldots, a_{s}$ th failures are observed, the rest are unobserved or missing, where $a_{1}, a_{2}, \ldots, a_{s}$ are considered to be fixed.

Let us assume that the multiply type-II censored sample from a sample of size $n$ is

$$
X_{a_{1}: n} \leq X_{a_{2}: n} \leq \cdots \leq X_{a_{s}: n}
$$

where $1 \leq a_{1}<a_{2}<\cdots<a_{s} \leq n, a_{0}=0, a_{s+1}=n+1, F\left(x_{a_{0}: n}\right)=0$, and $F\left(x_{a_{s+1}: n}\right)=1$.

The likelihood function for the logistic distribution based on the multiply type-II censored sample (2.1) is given by

$$
L=\frac{n !}{\prod_{j=1}^{s+1}\left(a_{j}-a_{j-1}-1\right) !} \prod_{j=1}^{s+1}\left[F\left(z_{a_{j}: n}\right)-F\left(z_{a_{j-1}: n}\right)\right]^{a_{j}-a_{j-1}-1}\left(\frac{\pi}{\sigma \sqrt{3}}\right)^{s} \prod_{j=1}^{s} f\left(z_{a_{j}: n}\right)
$$

where $Z_{i: n}=\pi\left(X_{i: n}-\theta\right) /(\sigma \sqrt{3})$, and $f(z)$ and $F(z)$ are the pdf and the cdf of the standard logistic distribution, respectively.

Since $f(z)=F(z)\{1-F(z)\}$, we can obtain the likelihood equations as follows;

$$
\begin{aligned}
\frac{\partial \ln L}{\partial \theta}= & -\frac{\pi}{\sigma \sqrt{3}}\left[s+\left(a_{1}-1\right)-\left(a_{1}-1\right) F\left(z_{a_{1}: n}\right)-\left(n-a_{s}\right) F\left(z_{a_{s}: n}\right)-2 \sum_{j=1}^{s} F\left(z_{a_{1}: n}\right)\right. \\
& \left.+\sum_{j=2}^{s}\left(a_{j}-a_{j-1}-1\right) \frac{f\left(z_{a_{j}: n}\right)-f\left(z_{a_{j-1}: n}\right)}{F\left(z_{a_{j}: n}\right)-F\left(z_{a_{j-1}: n}\right)}\right] \\
= & 0
\end{aligned}
$$

and

$$
\frac{\partial \ln L}{\partial \sigma}=-\frac{1}{\sigma}\left[s+\left(a_{1}-1\right) z_{a_{1}: n}-\left(a_{1}-1\right) F\left(z_{a_{1}: n}\right) z_{a_{1}: n}\right.
$$




$$
\begin{aligned}
& \quad-\left(n-a_{s}\right) F\left(z_{a_{s}: n}\right) z_{a_{s}: n}+\sum_{j=1}^{s} z_{a_{j}: n}-2 \sum_{j=1}^{s} F\left(z_{a_{1}: n}\right) z_{a_{j}: n} \\
& \left.\quad+\sum_{j=2}^{s}\left(a_{j}-a_{j-1}-1\right) \frac{f\left(z_{a_{j}: n}\right) z_{a_{j}: n}-f\left(z_{a_{j-1}: n}\right) z_{a_{j-1}: n}}{F\left(z_{a_{j}: n}\right)-F\left(z_{a_{j-1}: n}\right)}\right] \\
& =0 .
\end{aligned}
$$

Since the likelihood equations are very complicated, the equations (2.4) and (2.5) do not admit explicit solutions for $\mu$ and $\theta$, respectively.

Let $\xi_{i}=F^{-1}\left(p_{i}\right)=\ln \left[p_{i} /\left(1-p_{i}\right)\right]$, where $p_{i}=i /(n+1), q_{i}=1-p_{i}$.

First, the equation (2.4) does not admit an explicit solution for $\theta$. But we can expand the following function by Taylor series expansion as follows;

$$
F\left(z_{a_{j}: n}\right) \simeq \kappa_{1 j}+\delta_{1 j} z_{a_{j}: n}
$$

and

$$
\frac{f\left(z_{a_{j}: n}\right)-f\left(z_{a_{j-1}: n}\right)}{F\left(z_{a_{j}: n}\right)-F\left(z_{a_{j-1}: n}\right)} \simeq \alpha_{1 j}+\beta_{1 j} z_{a_{j}: n}+\gamma_{1 j} z_{a_{j-1}: n}
$$

where

$$
\begin{aligned}
\kappa_{1 j} & =p_{a_{j}}-p_{a_{j}} q_{a_{j}} \xi_{a_{j}}, \quad \delta_{1 j}=p_{a_{j}} q_{a_{j}}, \\
\alpha_{1 j} & =\frac{\left(1+K_{j}\right)\left(p_{a_{j}} q_{a_{j}}-p_{a_{j-1}} q_{a_{j-1}}\right)}{p_{a_{j}}-p_{a_{j-1}}}-\frac{\xi_{a_{j}} p_{a_{j}} q_{a_{j}}\left(1-2 p_{a_{j}}\right)-\xi_{a_{j-1}} p_{a_{j-1}} q_{a_{j-1}}\left(1-2 p_{a_{j-1}}\right)}{p_{a_{j}}-p_{a_{j-1}}}, \\
\beta_{1 j} & =\frac{1}{p_{a_{j}}-p_{a_{j-1}}}\left[p_{a_{j}} q_{a_{j}}\left(1-2 p_{a_{j}}\right)-\frac{\left(p_{a_{j}} q_{a_{j}}\right)^{2}}{p_{a_{j}}-p_{a_{j-1}}}+\frac{p_{a_{j}} q_{a_{j}} p_{a_{j-1}} q_{a_{j-1}}}{p_{a_{j}}-p_{a_{j-1}}}\right], \\
\gamma_{1 j} & =\frac{1}{p_{a_{j}}-p_{a_{j-1}}}\left[\frac{p_{a_{j-1}} q_{a_{j-1}}\left(p_{a_{j}} q_{a_{j}}-p_{a_{j-1}} q_{a_{j-1}}\right)}{p_{a_{j}}-p_{a_{j-1}}}-p_{a_{j-1}} q_{a_{j-1}}\left(1-2 p_{a_{j-1}}\right)\right], \\
K_{j} & =\frac{f\left(\xi_{a_{j}}\right) \xi_{a_{j}}-f\left(\xi_{a_{j-1}}\right) \xi_{a_{j-1}}}{p_{a_{j}}-p_{a_{j-1}}} .
\end{aligned}
$$

Next, we can approximate the following function by

$$
F\left(z_{a_{j}: n}\right) z_{a_{j}: n} \simeq \kappa_{2 j}+\delta_{2 j} z_{a_{j}: n}
$$

and

$$
\frac{f\left(z_{a_{j}: n}\right) z_{a_{j}: n}-f\left(z_{a_{j-1}: n}\right) z_{a_{j-1}: n}}{F\left(z_{a_{j}: n}\right)-F\left(z_{a_{j-1}: n}\right)} \simeq \alpha_{2 j}+\beta_{2 j} z_{a_{j}: n}+\gamma_{2 j} z_{a_{j-1}: n},
$$

where

$$
\begin{aligned}
\kappa_{2 j} & =-\xi_{a_{j}}^{2} p_{a_{j}} q_{a_{j}}, \quad \delta_{2 j}=\xi_{a_{j}} p_{a_{j}} q_{a_{j}}+p_{a_{j}} \\
\alpha_{2 j} & =K_{j}^{2}-\frac{\xi_{a_{j}} p_{a_{j}} q_{a_{j}}\left(1-2 p_{a_{j}}\right)-\xi_{a_{j-1}} p_{a_{j-1}} q_{a_{j-1}}\left(1-2 p_{a_{j-1}}\right)}{p_{a_{j}}-p_{a_{j-1}}} \\
\beta_{2 j} & =\frac{\left(1-K_{j}\right) p_{a_{j}} q_{a_{j}}+\xi_{a_{j}} p_{a_{j}} q_{a_{j}}\left(1-2 p_{a_{j}}\right)}{p_{a_{j}}-p_{a_{j-1}}} \\
\gamma_{2 j} & =-\frac{\left(1-K_{j}\right) p_{a_{j-1}} q_{a_{j-1}}+\xi_{a_{j-1}} p_{a_{j-1}} q_{a_{j-1}}\left(1-2 p_{a_{j-1}}\right)}{p_{a_{j}}-p_{a_{j-1}}}
\end{aligned}
$$


By substituting the equations (2.5) and (2.6) into the equation (2.4), and the equations (2.7) and (2.8) into the equation (2.5), we can derive an estimator of $\theta$ as follows;

$$
\hat{\theta}_{1}=\frac{B_{0} A_{1}-A_{0} C_{1}}{C_{0} A_{1}-A_{0} B_{1}}
$$

where

$$
\begin{aligned}
A_{0}= & s+\left(a_{1}-1\right)-\left(a_{1}-1\right) \kappa_{11}-\left(n-a_{s}\right) \kappa_{1 s}-2 \sum_{j=1}^{s} \kappa_{1 j}+\sum_{j=2}^{s}\left(a_{j}-a_{j-1}-1\right) \alpha_{1 j}, \\
B_{0}= & -\left(a_{1}-1\right) \delta_{11} X_{a_{1}: n}-\left(n-a_{s}\right) \delta_{1 s} X_{a_{s}: n}-2 \sum_{j=1}^{s} \delta_{1 j} X_{a_{j}: n} \\
& +\sum_{j=2}^{s}\left(a_{j}-a_{j-1}-1\right)\left(\beta_{1 j} X_{a_{j}: n}+\gamma_{1 j} X_{a_{j-1}: n}\right), \\
C_{0}= & -\left(a_{1}-1\right) \delta_{11}-\left(n-a_{s}\right) \delta_{1 s}-2 \sum_{j=1}^{s} \delta_{1 j}+\sum_{j=2}^{s}\left(a_{j}-a_{j-1}-1\right)\left(\beta_{1 j}+\gamma_{1 j}\right), \\
A_{1}= & s-\left(a_{1}-1\right) \kappa_{21}-\left(n-a_{s}\right) \kappa_{2 s}-2 \sum_{j=1}^{s} \kappa_{2 j}+\sum_{j=2}^{s}\left(a_{j}-a_{j-1}-1\right) \alpha_{2 j}, \\
B_{1}= & \left(a_{1}-1\right)\left(1-\delta_{21}\right)-\left(n-a_{s}\right) \delta_{2 s}+s-2 \sum_{j=1}^{s} \delta_{2 j}+\sum_{j=2}^{s}\left(a_{j}-a_{j-1}-1\right)\left(\beta_{2 j}+\gamma_{2 j}\right), \\
C_{1}= & \left(a_{1}-1\right)\left(1-\delta_{21}\right) X_{a_{1}: n}-\left(n-a_{s}\right) \delta_{2 s} X_{a_{s}: n}+\sum_{j=1}^{s} X_{a_{j}: n}-2 \sum_{j=1}^{s} \delta_{2 j} X_{a_{j}: n} \\
& +\sum_{j=2}^{s}\left(a_{j}-a_{j-1}-1\right)\left(\beta_{2 j} X_{a_{j}: n}+\gamma_{2 j} X_{a_{j-1}: n}\right) .
\end{aligned}
$$

By substituting the equations (2.5) and (2.8) into the equation (2.5), we can derive an estimator of $\sigma$ as follows;

$$
\hat{\sigma}_{1}=\frac{\pi}{\sqrt{3}}\left(\frac{-B_{2}+\sqrt{B_{2}^{2}-4 A_{2} C_{2}}}{2 A_{2}}\right),
$$

where

$$
\begin{aligned}
A_{2}= & s+\sum_{j=2}^{s}\left(a_{j}-a_{j-1}-1\right) \alpha_{2 j} \\
B_{2}= & \left(a_{1}-1\right)\left(X_{a_{1}: n}-\hat{\theta}\right)\left(1-\kappa_{11}\right)-\left(n-a_{s}\right) \kappa_{11}\left(X_{a_{s}: n}-\hat{\theta}\right)+\sum_{j=1}^{s}\left(X_{a_{j}: n}-\hat{\theta}\right) \\
& \quad-2 \sum_{j=1}^{s} \kappa_{1 j}\left(X_{a_{j}: n}-\hat{\theta}\right)+\sum_{j=2}^{s}\left(a_{j}-a_{j-1}-1\right)\left\{\beta_{2 j}\left(X_{a_{j}: n}-\hat{\theta}\right)+\gamma_{2 j}\left(X_{a_{j-1}: n}-\hat{\theta}\right)\right\}, \\
& \\
C_{2}= & -\left(a_{1}-1\right) \delta_{11}\left(X_{a_{1}: n}-\hat{\theta}\right)^{2}-\left(n-a_{s}\right) \delta_{11}\left(X_{a_{s}: n}-\hat{\theta}\right)^{2}-2 \sum_{j=1}^{s} \delta_{1 j}\left(X_{a_{j}: n}-\hat{\theta}\right)^{2} .
\end{aligned}
$$


It should be mentioned that upon solving equation (2.5) for $\sigma$, we obtain a quadratic equation in $\sigma$ that has two roots. However, one of them drops out, since $\delta_{1 j}>0$.

Balakrishnan et al. (1995) derived an AMLE of the location parameter $\theta$ and the scale parameter $\sigma$ as follows;

$$
\hat{\theta}_{2}=B_{3}-\frac{\pi}{\sqrt{3}} \hat{\sigma}_{2} C_{3}
$$

and

$$
\hat{\sigma}_{2}=\frac{\pi}{\sqrt{3}}\left(\frac{-D+\sqrt{D^{2}+4 s E}}{2 s}\right),
$$

where

$$
\begin{aligned}
& B_{3}=\frac{1}{g}\left[\left(a_{1}-1\right) \delta_{11} X_{a_{1}: n}+\left(n-a_{s}\right) \delta_{1 s} X_{a_{s}: n}+2 \sum_{j=1}^{s} \delta_{1 j} X_{a_{j}: n}\right. \\
&\left.+\sum_{j=2}^{s}\left(a_{j}-a_{j-1}-1\right)\left(\delta_{1 j} X_{a_{j}: n}+\delta_{1 j-1} X_{a_{j-1}: n}\right)\right] \\
& C_{3}= \frac{1}{g}\left[s+\left(a_{1}-1\right)\left(1-\kappa_{11}\right)-\left(n-a_{s}\right) \kappa_{1 s}+2 \sum_{j=1}^{s} \kappa_{1 j}+\sum_{j=2}^{s}\left(a_{j}-a_{j-1}-1\right) L_{j}\right], \\
& D=\left(a_{1}-1\right)\left(1-\kappa_{11}\right) X_{a_{1}: n}-\left(n-a_{s}\right) \kappa_{1 s} X_{a_{s}: n}+2 \sum_{j=1}^{s}\left(1-2 \kappa_{1 j}\right) X_{a_{j}: n} \\
&+\sum_{j=2}^{s}\left(a_{j}-a_{j-1}-1\right) L_{j}^{*}-g B_{3} C_{3}, \\
& E=\left(a_{1}-1\right) \delta_{11}\left(X_{a_{1}: n}-B_{3}\right)^{2}+\left(n-a_{s}\right) \delta_{1 s}\left(X_{a_{s}: n}-B_{3}\right)^{2}+2 \sum_{j=1}^{s} \delta_{1 j}\left(X_{a_{j}: n}-B_{3}\right)^{2}, \\
&+\sum_{j=2}^{s}\left(a_{j}-a_{j-1}-1\right) \frac{p_{a_{j}} q_{a_{j}} p_{a_{j-1}} q_{a_{j-1}}\left(x_{a_{j}: n}-X_{a_{j-1}}\right)^{2}}{\left(p_{a_{j-1}: n}\right)^{2},} \\
& g=\left(a_{1}-1\right) \delta_{11}+\left(n-a_{s}\right) \delta_{1 s}+2 \sum_{j=1}^{s} \delta_{1 j}+\sum_{j=2}^{s}\left(a_{j}-a_{j-1}-1\right)\left(\delta_{1 j-1}-\delta_{1 j}\right), \\
& L_{j}= \delta_{1 j}\left[\left(p_{a_{j}}-p_{a_{j-1}}\right)^{-1}+\xi_{a_{j}}\right]-\delta_{1 j-1}\left[\left(p_{a_{j}}-p_{a_{j-1}}\right)^{-1}+\xi_{a_{j-1}}\right], \\
& L_{j}^{*}= \delta_{1 j}\left[\left(p_{a_{j}}-p_{a_{j-1}}\right)^{-1}+\xi_{a_{j}}\right] X_{a_{j}: n}-\delta_{1 j-1}\left[\left(p_{a_{j}}-p_{a_{j-1}}\right)^{-1}+\xi_{a_{j-1}}\right] X_{a_{j-1}: n .} .
\end{aligned}
$$

\section{Goodness of fit tests}

In this section, we consider some goodness-of-fit tests of the logistic distribution based on multiply type-II censored samples. 


\subsection{Modified empirical distribution function type tests}

A well known $\operatorname{EDF} F_{n}(x)$ is

$$
F_{n}(x)=\frac{\text { the number of } X^{\prime} s \leq x}{n} .
$$

For complete samples under a simple hypothesis, the Kolmogorov-Smirnov $(D)$, the Cramervon Mises $\left(W^{2}\right)$, the Anderson-Darling $\left(A^{2}\right)$, and the Watson statistics $\left(U^{2}\right)$ are defined as

$$
\begin{aligned}
& D^{+}=\sup _{x}\left[F_{n}(x)-F_{0}(x)\right], \\
& D^{-}=\sup _{x}\left[F_{0}(x)-F_{n}(x)\right], \\
& D=\max \left[D^{+}, D^{-}\right], \\
& W^{2}=n \int_{-\infty}^{\infty}\left[F_{n}(x)-F_{0}(x)\right]^{2} d F_{0}(x), \\
& A^{2}=n \int_{-\infty}^{\infty} \frac{\left[F_{n}(x)-F_{0}(x)\right]^{2}}{F_{0}(x)\left\{1-F_{0}(x)\right\}} d F_{0}(x),
\end{aligned}
$$

and

$$
U^{2}=W^{2}-n\left(\bar{F}_{n}(x)-\frac{1}{2}\right)^{2},
$$

where $F_{0}(x)$ is the cdf assumed under $H_{0}$ and $\bar{F}_{n}(x)$ is the mean of the $F_{n}(x)$.

In the form given above, test statistics can only be used with complete samples, i.e. no censoring. Modification of the test statistics for censored samples and for composite hypothesis $H_{0}$ with unspecified parameters has been studied by Pettitt and Stephens (1976). Kang and Lee (2006) developed three EDF type modified Kolmogorov-Smirnov test, modified Anderson-Darling test, and modified Cramer-von Mises test for the two-parameter exponential distribution based on multiply type-II censored samples.

Now, we use three EDF type modified Kolmogorov-Smirnov test, modified AndersonDarling test, and modified Cramer-von Mises test for multiply type-II censored samples from the logistic distribution using the proposed estimators $\hat{\sigma}_{k}$ and $\hat{\theta}_{k}, k=1,2$ as follows;

$$
\begin{aligned}
D_{k}^{+} & =\max _{1 \leq a_{j} \leq s}\left[\frac{a_{j}}{s}-F\left(x_{a_{j}: n} ; \hat{\sigma}_{k}, \hat{\theta}_{k}\right)\right], \\
D_{k}^{-} & =\max _{1 \leq a_{j} \leq s}\left[F\left(x_{a_{j}: n} ; \hat{\sigma}_{k}, \hat{\theta}_{k}\right)-\frac{a_{j}}{s}\right], \\
D_{k} & =\max _{1 \leq a_{j} \leq s}\left[D_{k}^{+}, D_{k}^{-}\right], \\
W_{k}^{2} & =\frac{1}{12 s}+\sum_{j=1}^{s}\left[F\left(x_{a_{j}: n} ; \hat{\sigma}_{k}, \hat{\theta}_{k}\right)-\frac{2 a_{j}-1}{2 s}\right]^{2},
\end{aligned}
$$


and

$$
A_{k}^{2}=-s-\frac{1}{s}+\sum_{j=1}^{s}\left(2 a_{j}-1\right)\left[\ln F\left(x_{a_{j}: n} ; \hat{\sigma}_{k}, \hat{\theta}_{k}\right)+\ln \left\{1-F\left(x_{a_{s+1-j}: n} ; \hat{\sigma}_{k}, \hat{\theta}_{k}\right)\right\}\right] .
$$

We also obtained the modified Watson test for multiply type-II censored samples from the logistic distribution using the proposed estimators $\hat{\sigma}_{k}$ and $\hat{\theta}_{k}$ as follows;

$$
U_{k}^{2}=W_{k}^{2}-s\left(\frac{1}{s} \sum_{j=1}^{s} F\left(x_{a_{j}: n} ; \hat{\sigma}_{k}, \hat{\theta}_{k}\right)-\frac{1}{2}\right)^{2} .
$$

\subsection{Modified normalized sample Lorenz curve}

The Lorenz curve is extensively used in the study of income distribution and used to be a powerful tool for the analysis of a variety of scientific problems.

Cho et al. (1999) proposed the transformed Lorenz curve that can be used in the study of symmetric distribution. The transformed Lorenz curve is defined by

$$
T L\left(r_{i}\right)=\frac{\sum_{j=1}^{i} X_{j: n}}{\sum_{j=1}^{n} X_{j: n}}, \quad r_{i}=\frac{i}{n}, \quad i=1,2, \ldots, n .
$$

Kang and Cho (2001) proposed the NSLC for the complete sample as follows;

$$
N S L C\left(r_{i}\right)=\frac{T S L\left(r_{i}\right)}{T S L_{F}\left(r_{i}\right)}, \quad r_{i}=\frac{i}{n}, \quad i=1,2, \ldots, n,
$$

where

$$
\begin{aligned}
\operatorname{TSL}\left(r_{i}\right) & =\frac{\sum_{j=1}^{i}\left(X_{j: n}-X_{1: n}\right)}{\sum_{j=1}^{n}\left(X_{j: n}-X_{1: n}\right)}-r_{i}+1, \\
T S L_{F}\left(r_{i}\right) & =\frac{\sum_{j=1}^{i}\left[F^{-1}\left(p_{j}\right)-F^{-1}\left(p_{1}\right)\right]}{\sum_{j=1}^{n}\left[F^{-1}\left(p_{j}\right)-F^{-1}\left(p_{1}\right)\right]}-r_{i}+1 .
\end{aligned}
$$

Now, we propose modified NSLC based on multiply type-II censored samples.

The modified NSLC based on multiply type-II censored samples is given by

$$
\operatorname{MNSLC}\left(r_{i}\right)=\frac{\operatorname{MTSL}\left(r_{i}\right)}{T S L_{F}\left(r_{i}\right)}, \quad r_{i}=\frac{a_{i}}{n}, \quad i=1,2, \ldots, s,
$$

where

$$
\operatorname{MTSL}\left(r_{i}\right)=\frac{\sum_{j=1}^{i}\left(X_{a_{j}: n}-X_{a_{1}: n}\right)}{\sum_{j=1}^{s}\left(X_{a_{j}: n}-X_{a_{1}: n}\right)}-r_{i}+1 .
$$

We also propose the modified NSLC plot for multiply type-II censored samples using $(\mathrm{X}, \mathrm{Y})=\left(1-r_{i}, 1-M N S L C\left(r_{i}\right)\right)$. If data come from the logistic distribution, the modified NSLC plot is $y=0$ (see, Figure 3.1 and Figure 3.2). The value of $1-M N S L C\left(r_{i}\right)$ increases 
and then decreases as $1-r_{i}$ increases for all alternative distributions. The modified NSLC plot is a left skewed form when the alternative distributions are the lognormal (LN), exponential (Exp), and Rayleigh distributions (Rayleigh). However, the modified NSLC plot is a symmetrical form when the alternative distribution is the double Rayleigh (DR) distribution.

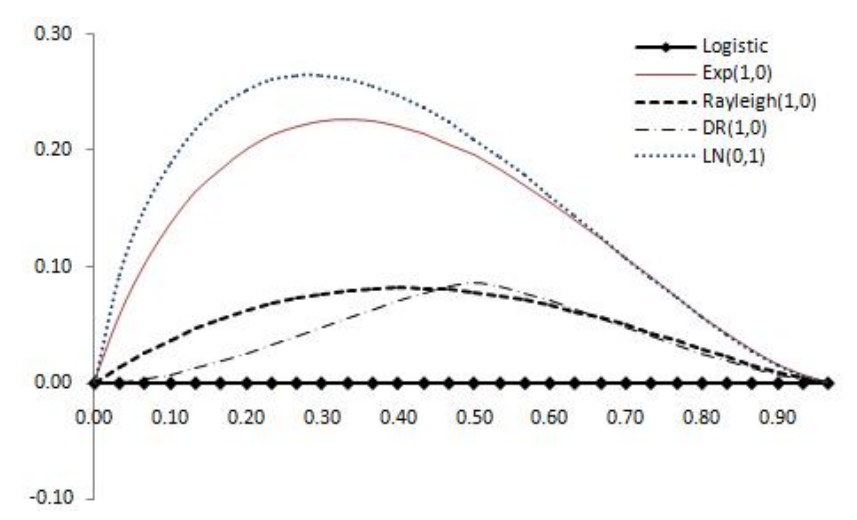

Figure 3.1 Modified NSLC plot : Complete data $(n=30, m=0)$

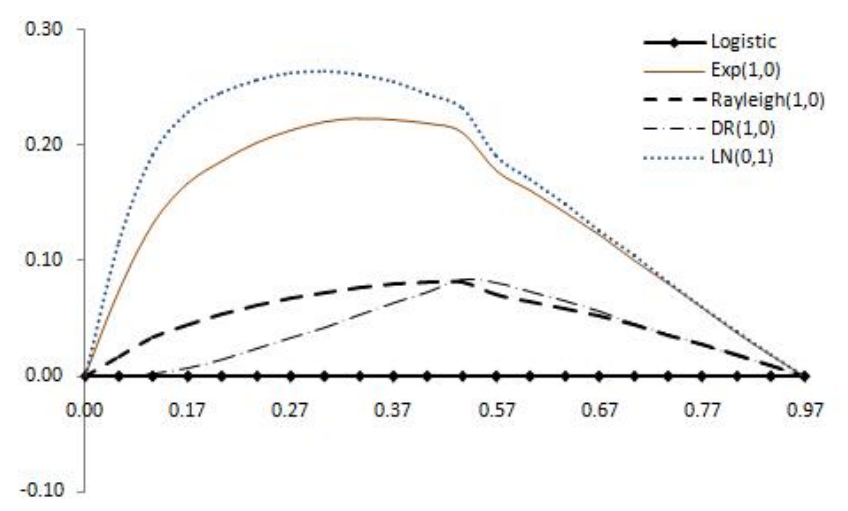

Figure 3.2 Modified NSLC plot : Multiply type-II censored data $\left(n=30, a_{j}=1,5 \sim 13,17 \sim 25,28 \sim 30\right)$

\section{Simulation study and illustrative example}

In order to evaluate the performance of the proposed estimators, the mean squared errors of all proposed estimators were simulated by a Monte Carlo method for sample size $n=20,40$ and various choices of censoring ( $m=n-s$ is the number of unobserved or missing data). The simulation procedure was repeated 10,000 times.

From Table $4.2, \hat{\theta}_{1}$ and $\hat{\sigma}_{1}$ are more efficient than $\hat{\theta}_{2}$ and $\hat{\sigma}_{2}$ in the sense of the MSE. As expected, the MSEs of all estimators decreases as sample size $n$ increases. For fixed sample size, the mean squared errors increases generally as $m$ increases. 


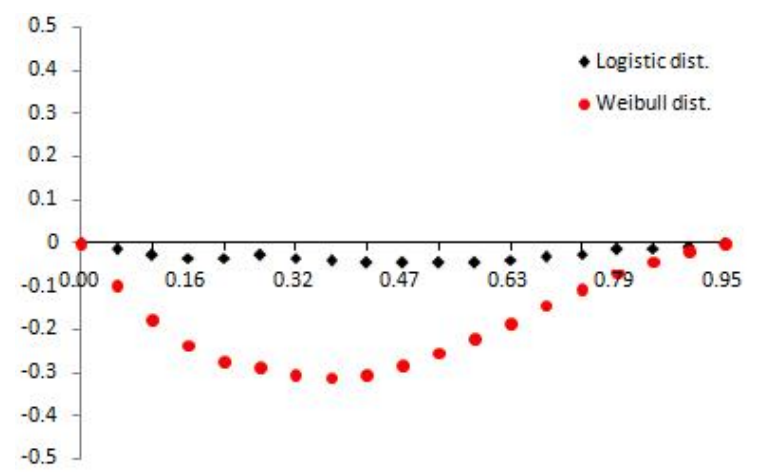

Figure 4.1 Modified NSLC plot : Complete data $(n=19, m=0)$

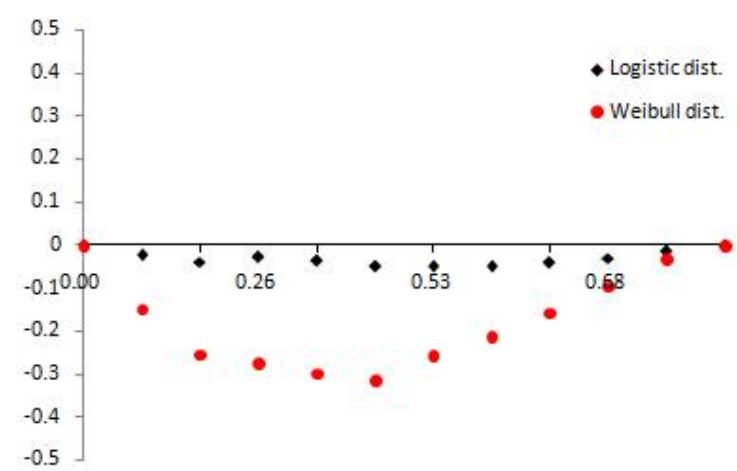

Figure 4.2 Modified NSLC plot : Multiply type-II censored data $\left(n=19, a_{j}=1,2,6 \sim 9,12 \sim 15,18,19\right)$

In order to evaluate the performance of the proposed goodness-of-fit tests, the powers were simulated by a Monte Carlo method for sample size $n=20,40$ and various choices of censoring. This simulation procedure was repeated 10,000 times. We compute critical value $(\alpha=0.05)$ and type I error for four tests under the logistic distribution (see Table $4.3 \sim$ 4.4).

The powers of five tests with significance level 0.05 for the logistic distribution based on multiply type-II censored samples are investigated under 4 alternative distributions. These values are presented in Table $4.5 \sim 4.8$.

The modified EDF type tests that use the estimators $\hat{\theta}_{1}$ and $\hat{\sigma}_{1}$ are generally more powerful than the tests that use the estimators $\hat{\theta}_{2}$ and $\hat{\sigma}_{2}$ when multiply type-II censored data.

For the exponential, lognormal, double Rayleigh alternative distribution, the modified Anderson-Darling test is more powerful than other tests. Especially, for the double Rayleigh alternative distribution, the modified Watson test showed good performance when complete data.

All computation were programmed in Microsoft Visual $\mathrm{C}++6.0$ and random numbers for simulations were generated by IMSL subroutines.

We present an example to illustrate the inference procedures discussed in this paper.

We apply the proposed graphical methods to a real data set. Let us consider the following data, which represent failure times to breakdown of an insulating fluid tested at 34 kilovolts. 
This data, taken from Table 6.1 in Nelson (1982);

$0.19,0.78,0.96,1.31,2.78,3.16,4.15,4.67,4.85,6.50,7.35,8.01,8.27,12.06,31.75,32.52$, $33.91,36.71,72.89$

This data had been utilized earlier by Kus and Kaya (2006). They showed that the loglogistic distribution provides better fits to Nelson's data than the Weibull distribution.

We apply those data, which represent failure log-lifetimes to breakdown of an insulating fluid tested at 34 kilovolts.

$-1.66,-0.25,-0.04,0.27,1.02,1.15,1.42,1.54,1.58,1.87,1.99,2.08,2.11,2.49,3.46,3.48$, $3.52,3.60,4.29$

For complete data, we can obtain the AMLEs $\hat{\sigma}_{1}=0.865, \hat{\theta}_{1}=1.834$ and $\hat{\sigma}_{2}=0.865$, $\hat{\theta}_{2}=1.834$. For this example of $n=19, s=12\left(a_{j}=1,2,6 \sim 9,12 \sim 15,18,19\right)$, and the multiply type-II censored samples are -1.66, -0.25, -, -, -, 1.15, 1.42, 1.54, 1.58, -, -, 2.08, $2.11,2.49,3.46,-,-, 3.60,4.29$, we can obtain the AMLEs $\hat{\sigma}_{1}=0.846, \hat{\theta}_{1}=1.847$, and $\hat{\sigma}_{2}=1.276, \hat{\theta}_{2}=1.714$.

We can picture the proposed plots for multiply type-II censored samples. It is easy to see that the modified NSLC plot is good performance when cdf of $T S L_{F}\left(r_{i}\right)$ is the Logistic distribution (see, Figure 4.1 and Figure 4.2).

We also compute critical value $(\alpha=0.05)$ for compete data and the multiply type-II censored sample (see Table 4.1).

So, test statistics can be computed by (3.6), (3.7), (3.8), and (3.9) which provides strong evidence that the real data set is from a logistic distribution (Accept $H_{0}$ ).

Table 4.1 Critical value and proposed test statistics

\begin{tabular}{lcccccccc}
\hline \hline & $D_{1}$ & $D_{2}$ & $A_{1}^{2}$ & $A_{2}^{2}$ & $W_{1}^{2}$ & $W_{2}^{2}$ & $U_{1}^{2}$ & $U_{2}^{2}$ \\
\hline Critical Value & & & & & & & & \\
Compete data : & 0.167 & 0.167 & 0.658 & 0.658 & 0.103 & 0.103 & 0.103 & 0.103 \\
Censored sample : & 0.687 & 0.782 & 8.348 & 8.829 & 1.639 & 1.988 & 1.637 & 1.987 \\
\hline Test Statistics & & & & & & & & \\
Compete data : & 0.105 & 0.105 & 0.269 & 0.269 & 0.037 & 0.037 & 0.037 & 0.037 \\
Censored sample : & 0.636 & 0.701 & 7.958 & 8.441 & 1.546 & 1.716 & 1.544 & 1.708 \\
\hline
\end{tabular}

Table 4.2 The relative mean squared errors (biases) for the estimators of the location parameter $\theta$ and the scale parameter $\sigma$

\begin{tabular}{|c|c|c|c|c|c|c|}
\hline & & & $\hat{\theta}_{1}$ & $\hat{\sigma}_{1}$ & $\hat{\theta}_{2}$ & $\hat{\sigma}_{2}$ \\
\hline$n$ & $m$ & $a_{j}$ & MSE (bias) & MSE (bias) & MSE (bias) & MSE (bias) \\
\hline \multirow{10}{*}{20} & 0 & $1 \sim 20$ & $0.150(0.005)$ & $0.036(-0.006)$ & $0.150(0.005)$ & $0.036(-0.006)$ \\
\hline & \multirow{4}{*}{4} & $2 \sim 17$ & $0.150(-0.005)$ & $0.044(-0.030)$ & $0.150(-0.004)$ & $0.044(-0.030)$ \\
\hline & & $4 \sim 20$ & $0.151(0.016)$ & $0.044(-0.031)$ & $0.151(0.015)$ & $0.044(-0.031)$ \\
\hline & & $3 \sim 18$ & $0.150(0.006)$ & $0.044(-0.031)$ & $0.150(0.006)$ & $0.044(-0.031)$ \\
\hline & & $1 \sim 14 \quad 17 \quad 18$ & $0.150(-0.004)$ & $0.040(-0.018)$ & $0.152(0.005)$ & $0.062(0.101)$ \\
\hline & \multirow{3}{*}{5} & $3 \sim 17$ & $0.151(0.000)$ & $0.047(-0.037)$ & $0.151(0.001)$ & $0.047(-0.037)$ \\
\hline & & $4 \sim 18$ & $0.151(0.011)$ & $0.047(-0.038)$ & $0.151(0.011)$ & $0.047(-0.038)$ \\
\hline & & $1 \sim 13 \quad 16 \quad 17$ & $0.151(-0.010)$ & $0.042(-0.022)$ & $0.153(0.002)$ & $0.063(0.094)$ \\
\hline & \multirow{2}{*}{10} & $126 \sim 10 \quad 13 \sim 15$ & $0.155(-0.021)$ & $0.050(-0.030)$ & $0.157(-0.027)$ & $0.308(0.435)$ \\
\hline & & $1 \sim 5 \quad 11 \sim 15$ & $0.158(-0.021)$ & $0.048(-0.031)$ & $0.174(-0.103)$ & $0.278(0.412)$ \\
\hline \multirow{9}{*}{40} & 0 & $1 \sim 40$ & $0.075(0.001)$ & $0.018(-0.003)$ & $0.075(0.001)$ & $0.018(-0.003)$ \\
\hline & \multirow{4}{*}{4} & $2 \sim 37$ & $0.075(-0.002)$ & $0.020(-0.009)$ & $0.075(-0.001)$ & $0.020(-0.009)$ \\
\hline & & $4 \sim 39$ & $0.075(0.003)$ & $0.020(-0.009)$ & $0.075(0.003)$ & $0.020(-0.009)$ \\
\hline & & $3 \sim 38$ & $0.075(0.001)$ & $0.020(-0.010)$ & $0.075(0.001)$ & $0.020(-0.010)$ \\
\hline & & $1 \sim 20 \quad 23 \sim 30 \quad 33 \sim 40$ & $0.075(0.001)$ & $0.018(-0.003)$ & $0.075(0.005)$ & $0.034(0.101)$ \\
\hline & \multirow{3}{*}{5} & $3 \sim 37$ & $0.075(-0.000)$ & $0.020(-0.011)$ & $0.075(-0.000)$ & $0.020(-0.011)$ \\
\hline & & $4 \sim 38$ & $0.075(0.002)$ & $0.020(-0.011)$ & $0.075(0.002)$ & $0.020(-0.011)$ \\
\hline & & $1 \sim 24 \quad 27 \sim 37$ & $0.075(-0.003)$ & $0.019(-0.008)$ & $0.075(-0.002)$ & $0.024(0.041)$ \\
\hline & 15 & $1 \sim 10 \quad 16 \sim 20 \quad 26 \sim 35$ & $0.075(-0.005)$ & $0.020(-0.011)$ & $0.081(-0.056)$ & $0.123(0.294)$ \\
\hline
\end{tabular}


Table 4.3 Critical values $(\alpha=0.05)$

\begin{tabular}{|c|c|c|c|c|c|c|c|c|c|c|}
\hline$n$ & $m$ & $a_{j}$ & $D_{1}$ & $D_{2}$ & $A_{1}^{2}$ & $A_{2}^{2}$ & $W_{1}^{2}$ & $W_{2}^{2}$ & $U_{1}^{2}$ & $U_{2}^{2}$ \\
\hline \multirow{10}{*}{20} & 0 & $1 \sim 20$ & 0.163 & 0.163 & 0.660 & 0.660 & 0.102 & 0.102 & 0.102 & 0.102 \\
\hline & \multirow{4}{*}{4} & $2 \sim 17$ & 0.321 & 0.321 & 4.506 & 4.511 & 0.371 & 0.373 & 0.323 & 0.324 \\
\hline & & $4 \sim 20$ & 0.348 & 0.348 & 11.378 & 11.382 & 0.497 & 0.495 & 0.473 & 0.470 \\
\hline & & $3 \sim 18$ & 0.334 & 0.334 & 7.643 & 7.643 & 0.427 & 0.427 & 0.425 & 0.425 \\
\hline & & $1 \sim 14 \quad 17 \quad 18$ & 0.332 & 0.352 & 1.492 & 1.727 & 0.345 & 0.398 & 0.222 & 0.279 \\
\hline & \multirow{3}{*}{5} & $3 \sim 17$ & 0.382 & 0.383 & 7.644 & 7.645 & 0.586 & 0.587 & 0.568 & 0.569 \\
\hline & & $4 \sim 18$ & 0.401 & 0.401 & 10.935 & 10.936 & 0.682 & 0.681 & 0.680 & 0.678 \\
\hline & & $1 \sim 13 \quad 16 \quad 17$ & 0.383 & 0.399 & 1.908 & 2.112 & 0.455 & 0.508 & 0.267 & 0.327 \\
\hline & \multirow{2}{*}{10} & $126 \sim 10 \quad 13 \sim 15$ & 0.827 & 0.888 & 7.789 & 8.217 & 2.222 & 2.306 & 2.111 & 2.235 \\
\hline & & $1 \sim 5 \quad 11 \sim 15$ & 0.824 & 0.865 & 4.904 & 5.254 & 2.234 & 2.293 & 2.075 & 2.202 \\
\hline \multirow{9}{*}{40} & 0 & $1 \sim 40$ & 0.116 & 0.116 & 0.660 & 0.660 & 0.099 & 0.099 & 0.099 & 0.099 \\
\hline & \multirow{4}{*}{4} & $2 \sim 37$ & 0.190 & 0.191 & 4.628 & 4.629 & 0.239 & 0.240 & 0.216 & 0.216 \\
\hline & & $4 \sim 39$ & 0.194 & 0.194 & 11.838 & 11.838 & 0.268 & 0.268 & 0.249 & 0.249 \\
\hline & & $3 \sim 38$ & 0.192 & 0.192 & 8.037 & 8.037 & 0.255 & 0.255 & 0.254 & 0.254 \\
\hline & & $1 \sim 20 \quad 23 \sim 30 \quad 33 \sim 40$ & 0.197 & 0.216 & 1.898 & 2.054 & 0.253 & 0.321 & 0.244 & 0.311 \\
\hline & \multirow{3}{*}{5} & $3 \sim 37$ & 0.215 & 0.215 & 8.062 & 8.062 & 0.331 & 0.331 & 0.324 & 0.324 \\
\hline & & $4 \sim 38$ & 0.217 & 0.217 & 11.561 & 11.562 & 0.353 & 0.353 & 0.348 & 0.348 \\
\hline & & $1 \sim 2427 \sim 37$ & 0.216 & 0.224 & 1.688 & 1.810 & 0.305 & 0.338 & 0.235 & 0.267 \\
\hline & 15 & $1 \sim 10 \quad 16 \sim 20 \quad 26 \sim 35$ & 0.591 & 0.640 & 8.012 & 8.901 & 2.675 & 2.937 & 2.567 & 2.868 \\
\hline
\end{tabular}

Table 4.4 The probabilities of type I error for four tests under the logistic distribution

\begin{tabular}{|c|c|c|c|c|c|c|c|c|c|c|}
\hline $\bar{~} \bar{n}$ & $m$ & $\overline{a_{j}}$ & $\overline{D_{1}}$ & $\overline{D_{2}}$ & $A_{1}^{2}$ & $A_{2}^{2}$ & $W_{1}^{2}$ & $W_{2}^{2}$ & $\overline{\overline{U_{1}^{2}}}$ & $\overline{\overline{U_{2}^{2}}}$ \\
\hline \multirow{10}{*}{20} & 0 & $1 \sim 20$ & 0.047 & 0.047 & 0.051 & 0.051 & 0.050 & 0.050 & 0.050 & 0.050 \\
\hline & \multirow{4}{*}{4} & $2 \sim 17$ & 0.048 & 0.048 & 0.048 & 0.048 & 0.046 & 0.046 & 0.047 & 0.046 \\
\hline & & $4 \sim 20$ & 0.050 & 0.050 & 0.046 & 0.046 & 0.043 & 0.043 & 0.043 & 0.043 \\
\hline & & $3 \sim 18$ & 0.052 & 0.052 & 0.047 & 0.047 & 0.049 & 0.049 & 0.049 & 0.049 \\
\hline & & $1 \sim 14 \quad 17 \quad 18$ & 0.054 & 0.053 & 0.049 & 0.047 & 0.048 & 0.050 & 0.046 & 0.047 \\
\hline & \multirow{3}{*}{5} & $3 \sim 17$ & 0.048 & 0.049 & 0.043 & 0.043 & 0.045 & 0.045 & 0.045 & 0.045 \\
\hline & & $4 \sim 18$ & 0.050 & 0.051 & 0.050 & 0.049 & 0.050 & 0.051 & 0.050 & 0.051 \\
\hline & & $1 \sim 13 \quad 16 \quad 17$ & 0.047 & 0.048 & 0.048 & 0.047 & 0.045 & 0.046 & 0.043 & 0.047 \\
\hline & \multirow{2}{*}{10} & $126 \sim 10 \quad 13 \sim 15$ & 0.053 & 0.048 & 0.051 & 0.047 & 0.045 & 0.047 & 0.046 & 0.049 \\
\hline & & $1 \sim 5 \quad 11 \sim 15$ & 0.051 & 0.051 & 0.047 & 0.051 & 0.049 & 0.048 & 0.049 & 0.049 \\
\hline \multirow{9}{*}{40} & 0 & $1 \sim 40$ & 0.054 & 0.054 & 0.054 & 0.054 & 0.055 & 0.055 & 0.056 & 0.056 \\
\hline & \multirow{4}{*}{4} & $2 \sim 37$ & 0.050 & 0.050 & 0.054 & 0.054 & 0.060 & 0.060 & 0.058 & 0.058 \\
\hline & & $4 \sim 39$ & 0.054 & 0.054 & 0.058 & 0.059 & 0.054 & 0.054 & 0.054 & 0.054 \\
\hline & & $3 \sim 38$ & 0.054 & 0.054 & 0.055 & 0.055 & 0.054 & 0.054 & 0.055 & 0.055 \\
\hline & & $1 \sim 20 \quad 23 \sim 30 \quad 33 \sim 40$ & 0.051 & 0.051 & 0.055 & 0.053 & 0.051 & 0.051 & 0.052 & 0.051 \\
\hline & \multirow{3}{*}{5} & $3 \sim 37$ & 0.053 & 0.052 & 0.052 & 0.052 & 0.058 & 0.058 & 0.057 & 0.058 \\
\hline & & $4 \sim 38$ & 0.056 & 0.056 & 0.056 & 0.056 & 0.055 & 0.055 & 0.057 & 0.057 \\
\hline & & $1 \sim 24 \quad 27 \sim 37$ & 0.051 & 0.052 & 0.052 & 0.055 & 0.060 & 0.058 & 0.058 & 0.056 \\
\hline & 15 & $1 \sim 10 \quad 16 \sim 20 \quad 26 \sim 35$ & 0.049 & 0.049 & 0.049 & 0.051 & 0.052 & 0.050 & 0.052 & 0.052 \\
\hline
\end{tabular}

Table 4.5 The powers of the tests for the exponential alternative distribution $\operatorname{Exp}(1)$

\begin{tabular}{|c|c|c|c|c|c|c|c|c|c|c|}
\hline$n$ & $m$ & $a_{j}$ & $D_{1}$ & $D_{2}$ & $A_{1}^{2}$ & $A_{2}^{2}$ & $W_{1}^{2}$ & $W_{2}^{2}$ & $U_{1}^{2}$ & $U_{2}^{2}$ \\
\hline \multirow{10}{*}{20} & 0 & $1 \sim 20$ & 0.449 & 0.449 & 0.694 & 0.694 & 0.588 & 0.588 & 0.590 & 0.590 \\
\hline & \multirow{4}{*}{4} & $2 \sim 17$ & 0.225 & 0.228 & 0.398 & 0.399 & 0.458 & 0.459 & 0.426 & 0.427 \\
\hline & & $4 \sim 20$ & 0.176 & 0.174 & 0.395 & 0.394 & 0.393 & 0.391 & 0.407 & 0.407 \\
\hline & & $3 \sim 18$ & 0.197 & 0.197 & 0.354 & 0.354 & 0.414 & 0.414 & 0.411 & 0.411 \\
\hline & & $1 \sim 14 \quad 17 \quad 18$ & 0.190 & 0.160 & 0.734 & 0.643 & 0.646 & 0.538 & 0.579 & 0.551 \\
\hline & \multirow{3}{*}{5} & $3 \sim 17$ & 0.172 & 0.172 & 0.326 & 0.327 & 0.394 & 0.395 & 0.381 & 0.381 \\
\hline & & $4 \sim 18$ & 0.145 & 0.144 & 0.285 & 0.284 & 0.363 & 0.363 & 0.367 & 0.366 \\
\hline & & $1 \sim 13 \quad 16 \quad 17$ & 0.146 & 0.127 & 0.735 & 0.624 & 0.624 & 0.516 & 0.542 & 0.521 \\
\hline & \multirow{2}{*}{10} & $126 \sim 10 \quad 13 \sim 15$ & 0.040 & 0.006 & 0.495 & 0.388 & 0.454 & 0.304 & 0.466 & 0.342 \\
\hline & & $1 \sim 5 \quad 11 \sim 15$ & 0.050 & 0.033 & 0.167 & 0.561 & 0.162 & 0.363 & 0.185 & 0.424 \\
\hline \multirow{9}{*}{40} & 0 & $1 \sim 40$ & 0.855 & 0.855 & 0.970 & 0.970 & 0.899 & 0.899 & 0.899 & 0.899 \\
\hline & \multirow{4}{*}{4} & $2 \sim 37$ & 0.426 & 0.427 & 0.799 & 0.800 & 0.813 & 0.813 & 0.801 & 0.801 \\
\hline & & $4 \sim 39$ & 0.391 & 0.390 & 0.884 & 0.884 & 0.761 & 0.761 & 0.781 & 0.781 \\
\hline & & $3 \sim 38$ & 0.413 & 0.413 & 0.842 & 0.842 & 0.782 & 0.782 & 0.780 & 0.780 \\
\hline & & $1 \sim 20 \quad 23 \sim 30 \quad 33 \sim 40$ & 0.385 & 0.314 & 0.985 & 0.974 & 0.884 & 0.831 & 0.889 & 0.843 \\
\hline & \multirow{3}{*}{5} & $3 \sim 37$ & 0.348 & 0.349 & 0.776 & 0.776 & 0.765 & 0.765 & 0.755 & 0.755 \\
\hline & & $4 \sim 38$ & 0.335 & 0.334 & 0.818 & 0.818 & 0.739 & 0.738 & 0.750 & 0.750 \\
\hline & & $1 \sim 24 \quad 27 \sim 37$ & 0.331 & 0.281 & 0.921 & 0.905 & 0.848 & 0.808 & 0.838 & 0.813 \\
\hline & 15 & $1 \sim 10 \quad 16 \sim 20 \quad 26 \sim 35$ & 0.020 & 0.007 & 0.428 & 0.846 & 0.191 & 0.571 & 0.253 & 0.631 \\
\hline
\end{tabular}


Table 4.6 The powers of the tests for the lognormal alternative distribution $\operatorname{LN}(0,1)$

\begin{tabular}{|c|c|c|c|c|c|c|c|c|c|c|}
\hline$n$ & $m$ & $a_{j}$ & $D_{1}$ & $D_{2}$ & $A_{1}^{2}$ & $A_{2}^{2}$ & $W_{1}^{2}$ & $W_{2}^{2}$ & $U_{1}^{2}$ & $U_{2}^{2}$ \\
\hline \multirow{10}{*}{20} & 0 & $1 \sim 20$ & 0.667 & 0.667 & 0.853 & 0.853 & 0.776 & 0.776 & 0.777 & 0.777 \\
\hline & \multirow{4}{*}{4} & $2 \sim 17$ & 0.308 & 0.309 & 0.517 & 0.518 & 0.573 & 0.575 & 0.546 & 0.547 \\
\hline & & $4 \sim 20$ & 0.338 & 0.336 & 0.586 & 0.586 & 0.609 & 0.608 & 0.621 & 0.620 \\
\hline & & $3 \sim 18$ & 0.314 & 0.314 & 0.492 & 0.492 & 0.582 & 0.582 & 0.581 & 0.581 \\
\hline & & $1 \sim 14 \quad 17 \quad 18$ & 0.317 & 0.279 & 0.830 & 0.744 & 0.760 & 0.670 & 0.706 & 0.682 \\
\hline & \multirow{3}{*}{5} & $3 \sim 17$ & 0.253 & 0.255 & 0.440 & 0.441 & 0.516 & 0.517 & 0.506 & 0.506 \\
\hline & & $4 \sim 18$ & 0.254 & 0.253 & 0.403 & 0.401 & 0.535 & 0.535 & 0.541 & 0.540 \\
\hline & & $1 \sim 13 \quad 16 \quad 17$ & 0.220 & 0.200 & 0.794 & 0.710 & 0.728 & 0.621 & 0.645 & 0.616 \\
\hline & \multirow{2}{*}{10} & $126 \sim 10 \quad 13 \sim 15$ & 0.056 & 0.008 & 0.530 & 0.416 & 0.507 & 0.346 & 0.517 & 0.378 \\
\hline & & $1 \sim 5 \quad 11 \sim 15$ & 0.073 & 0.030 & 0.211 & 0.572 & 0.203 & 0.383 & 0.232 & 0.449 \\
\hline \multirow{9}{*}{40} & 0 & $1 \sim 40$ & 0.957 & 0.957 & 0.994 & 0.994 & 0.976 & 0.976 & 0.976 & 0.976 \\
\hline & \multirow{4}{*}{4} & $2 \sim 37$ & 0.638 & 0.640 & 0.918 & 0.918 & 0.923 & 0.924 & 0.918 & 0.919 \\
\hline & & $4 \sim 39$ & 0.687 & 0.686 & 0.970 & 0.970 & 0.926 & 0.926 & 0.936 & 0.936 \\
\hline & & $3 \sim 38$ & 0.663 & 0.663 & 0.943 & 0.943 & 0.921 & 0.921 & 0.921 & 0.921 \\
\hline & & $1 \sim 20 \quad 23 \sim 30 \quad 33 \sim 40$ & 0.724 & 0.677 & 0.997 & 0.995 & 0.977 & 0.965 & 0.977 & 0.967 \\
\hline & \multirow{3}{*}{5} & $3 \sim 37$ & 0.579 & 0.579 & 0.907 & 0.907 & 0.903 & 0.903 & 0.897 & 0.897 \\
\hline & & $4 \sim 38$ & 0.605 & 0.605 & 0.936 & 0.936 & 0.904 & 0.904 & 0.911 & 0.911 \\
\hline & & $1 \sim 24 \quad 27 \sim 37$ & 0.553 & 0.513 & 0.968 & 0.962 & 0.941 & 0.920 & 0.933 & 0.921 \\
\hline & 15 & $1 \sim 10 \quad 16 \sim 20 \quad 26 \sim 35$ & 0.070 & 0.027 & 0.630 & 0.914 & 0.379 & 0.717 & 0.462 & 0.768 \\
\hline
\end{tabular}

Table 4.7 The powers of the tests for the double Rayleigh alternative distribution $\operatorname{DR}(0,1)$

\begin{tabular}{|c|c|c|c|c|c|c|c|c|c|c|}
\hline$n$ & $m$ & $a_{j}$ & $D_{1}$ & $D_{2}$ & $A_{1}^{2}$ & $A_{2}^{2}$ & $W_{1}^{2}$ & $W_{2}^{2}$ & $U_{1}^{2}$ & $U_{2}^{2}$ \\
\hline \multirow{10}{*}{20} & 0 & $1 \sim 20$ & 0.297 & 0.297 & 0.372 & 0.372 & 0.445 & 0.445 & 0.448 & 0.448 \\
\hline & \multirow{4}{*}{4} & $2 \sim 17$ & 0.106 & 0.106 & 0.197 & 0.197 & 0.112 & 0.112 & 0.118 & 0.118 \\
\hline & & $4 \sim 20$ & 0.114 & 0.114 & 0.271 & 0.271 & 0.056 & 0.055 & 0.046 & 0.045 \\
\hline & & $3 \sim 18$ & 0.099 & 0.099 & 0.310 & 0.310 & 0.076 & 0.076 & 0.076 & 0.076 \\
\hline & & $1 \sim 14 \quad 17 \quad 18$ & 0.096 & 0.051 & 0.261 & 0.080 & 0.215 & 0.065 & 0.167 & 0.078 \\
\hline & \multirow{3}{*}{5} & $3 \sim 17$ & 0.088 & 0.089 & 0.246 & 0.246 & 0.083 & 0.084 & 0.084 & 0.084 \\
\hline & & $4 \sim 18$ & 0.102 & 0.101 & 0.349 & 0.348 & 0.050 & 0.050 & 0.049 & 0.048 \\
\hline & & $1 \sim 13 \quad 16 \quad 17$ & 0.070 & 0.047 & 0.305 & 0.139 & 0.252 & 0.114 & 0.171 & 0.122 \\
\hline & \multirow{2}{*}{10} & $126 \sim 10 \quad 13 \sim 15$ & 0.031 & 0.011 & 0.279 & 0.189 & 0.159 & 0.126 & 0.157 & 0.149 \\
\hline & & $1 \sim 5 \quad 11 \sim 15$ & 0.031 & 0.170 & 0.058 & 0.216 & 0.066 & 0.165 & 0.074 & 0.173 \\
\hline \multirow{9}{*}{40} & 0 & $1 \sim 40$ & 0.691 & 0.691 & 0.800 & 0.800 & 0.858 & 0.858 & 0.859 & 0.859 \\
\hline & \multirow{4}{*}{4} & $2 \sim 37$ & 0.250 & 0.251 & 0.570 & 0.570 & 0.413 & 0.413 & 0.436 & 0.437 \\
\hline & & $4 \sim 39$ & 0.263 & 0.263 & 0.634 & 0.634 & 0.446 & 0.445 & 0.436 & 0.436 \\
\hline & & $3 \sim 38$ & 0.256 & 0.256 & 0.710 & 0.710 & 0.411 & 0.411 & 0.415 & 0.415 \\
\hline & & $1 \sim 20 \quad 23 \sim 30 \quad 33 \sim 40$ & 0.250 & 0.233 & 0.679 & 0.460 & 0.614 & 0.300 & 0.573 & 0.299 \\
\hline & \multirow{3}{*}{5} & $3 \sim 37$ & 0.192 & 0.192 & 0.628 & 0.628 & 0.279 & 0.280 & 0.293 & 0.293 \\
\hline & & $4 \sim 38$ & 0.215 & 0.215 & 0.714 & 0.714 & 0.295 & 0.294 & 0.295 & 0.295 \\
\hline & & $1 \sim 24 \quad 27 \sim 37$ & 0.178 & 0.091 & 0.643 & 0.408 & 0.480 & 0.203 & 0.387 & 0.228 \\
\hline & 15 & $1 \sim 10 \quad 16 \sim 20 \quad 26 \sim 35$ & 0.047 & 0.178 & 0.048 & 0.418 & 0.003 & 0.272 & 0.003 & 0.297 \\
\hline
\end{tabular}

Table 4.8 The powers of the tests for the Rayleigh alternative distribution Rayleigh $(0,1)$

\begin{tabular}{|c|c|c|c|c|c|c|c|c|c|c|}
\hline$n$ & $m$ & $a_{j}$ & $D_{1}$ & $D_{2}$ & $A_{1}^{2}$ & $A_{2}^{2}$ & $W_{1}^{2}$ & $W_{2}^{2}$ & $U_{1}^{2}$ & $U_{2}^{2}$ \\
\hline \multirow{10}{*}{20} & 0 & $1 \sim 20$ & 0.102 & 0.102 & 0.099 & 0.099 & 0.088 & 0.088 & 0.088 & 0.088 \\
\hline & \multirow{4}{*}{4} & $2 \sim 17$ & 0.058 & 0.058 & 0.070 & 0.070 & 0.103 & 0.103 & 0.090 & 0.090 \\
\hline & & $4 \sim 20$ & 0.045 & 0.044 & 0.085 & 0.085 & 0.069 & 0.069 & 0.072 & 0.072 \\
\hline & & $3 \sim 18$ & 0.051 & 0.051 & 0.069 & 0.069 & 0.090 & 0.090 & 0.089 & 0.089 \\
\hline & & $1 \sim 14 \quad 17 \quad 18$ & 0.037 & 0.034 & 0.172 & 0.135 & 0.151 & 0.116 & 0.098 & 0.104 \\
\hline & \multirow{3}{*}{5} & $3 \sim 17$ & 0.051 & 0.051 & 0.068 & 0.069 & 0.097 & 0.097 & 0.092 & 0.092 \\
\hline & & $4 \sim 18$ & 0.043 & 0.044 & 0.072 & 0.072 & 0.083 & 0.083 & 0.083 & 0.083 \\
\hline & & $\begin{array}{llll}1 \sim 13 & 16 & 17\end{array}$ & 0.029 & 0.027 & 0.194 & 0.156 & 0.163 & 0.129 & 0.098 & 0.115 \\
\hline & \multirow{2}{*}{10} & $126 \sim 10 \quad 13 \sim 15$ & 0.014 & 0.004 & 0.103 & 0.071 & 0.134 & 0.091 & 0.131 & 0.091 \\
\hline & & $1 \sim 5 \quad 11 \sim 15$ & 0.016 & 0.045 & 0.037 & 0.168 & 0.059 & 0.135 & 0.060 & 0.146 \\
\hline \multirow{9}{*}{40} & 0 & $1 \sim 40$ & 0.148 & 0.148 & 0.202 & 0.202 & 0.152 & 0.152 & 0.153 & 0.153 \\
\hline & \multirow{4}{*}{4} & $2 \sim 37$ & 0.060 & 0.060 & 0.075 & 0.075 & 0.114 & 0.115 & 0.102 & 0.102 \\
\hline & & $4 \sim 39$ & 0.048 & 0.048 & 0.175 & 0.175 & 0.080 & 0.080 & 0.089 & 0.088 \\
\hline & & $3 \sim 38$ & 0.053 & 0.053 & 0.121 & 0.121 & 0.091 & 0.091 & 0.091 & 0.091 \\
\hline & & $1 \sim 20 \quad 23 \sim 30 \quad 33 \sim 40$ & 0.037 & 0.025 & 0.275 & 0.209 & 0.081 & 0.073 & 0.078 & 0.075 \\
\hline & \multirow{3}{*}{5} & $3 \sim 37$ & 0.047 & 0.047 & 0.091 & 0.091 & 0.105 & 0.105 & 0.098 & 0.098 \\
\hline & & $4 \sim 38$ & 0.042 & 0.042 & 0.144 & 0.144 & 0.087 & 0.087 & 0.094 & 0.094 \\
\hline & & $1 \sim 24 \quad 27 \sim 37$ & 0.037 & 0.029 & 0.133 & 0.137 & 0.108 & 0.097 & 0.084 & 0.082 \\
\hline & 15 & $1 \sim 10 \quad 16 \sim 20 \quad 26 \sim 35$ & 0.003 & 0.012 & 0.009 & 0.168 & 0.019 & 0.106 & 0.023 & 0.114 \\
\hline
\end{tabular}




\section{Conclusions}

In most cases of censored and truncated samples, the maximum likelihood method does not provide explicit estimators. So we discuss another method for the purpose of providing the explicit estimators. We use four modified EDF types test for the logistic distribution based on multiply type-II censored samples using AMLEs. We propose the modified NSLC plot to test for the logistic distribution based on multiply type-II censored samples

Based on the simulation study and illustrative example, the modified EDF type tests that use the estimators $\hat{\theta}_{1}$ and $\hat{\sigma}_{1}$ are generally more powerful than the tests that use the estimators $\hat{\theta}_{2}$ and $\hat{\sigma}_{2}$ when multiply type-II censored data. In addition we believe that the modified NSLC plot can be extended to cases with various censoring.

We will need further study on the various graphical methods or test statistics for testing the distributions based on multiply type-II censored samples.

\section{References}

Balakrishnan, N. (1989). Approximate MLE of the scale parameter of the Rayleigh distribution with censoring. IEEE Transactions on Reliability, 38, 355-357.

Balakrishnan, N., Kannan, N., Lin, C. T. and Wu, S. J. S. (2004). Inference for the extreme value distribution under progressive type-II censoring. Journal of Statistical Computation $\mathscr{G}$ Simulation, 74, 25-45.

Balakrishnan, N. and Kateri, M. (2008). On the maximum likelihood estimation of parameters of Weibull distribution based on complete and censored data. Statistics \& 8 Probability Letters, 78, 2971-2975.

Balakrishnan, N., Gupta, S. S., and Panchapakesan, S. (1995). Estimation of the mean and standard deviation of the logistic distribution based on multiply type-II censored samples. Statistics, 27, 127-142.

Birnbaum, A. and Dudman, J. (1963). Logistic order statistics. The Annals of Mathematical Statistics, 34 658-663.

Choulakian, V. and Stephens, M. A. (2001). Goodness-of-fit tests for the generalized Pareto distribution. Technometrics, 43, 478-484.

Cho, Y. S., Lee, J. Y. and Kang, S. B. (1999). A study on distribution based on the transformed Lorenz curve. The Korean Journal of Applied Statistics, 12, 153-163.

Fei, H., Kong, F. and Tang, Y. (1995). Estimation for two-parameter Weibull distribution and extreme-value distribution under multiply type-II censoring. Communications in Statistics-Theory and Methods, 24, 2087-2104.

Han, J. T. and Kang, S. B. (2008). Estimation for the double Rayleigh distribution based on multiply type-II censored samples. Communications of the Korean Statistical Society, 15, 367-378.

Kang, S. B., Cho, Y. S. and Han, J. T. (2008). Estimation for the half logistic distribution under progressive type-II censoring. Communications of the Korean Statistical Society, 15, 815-823.

Kang, S. B. and Cho, Y. S. (2001). A study on distribution based on the normalized sample Lorenz curve. The Korean Communications in Statistics, 8, 185-192.

Kang, S. B. and Lee, S. K. (2006). Test for the exponential distribution based on multiply type-II censored samples. The Korean Communications in Statistics, 13, 537-550.

Kim, Y., Kang, S. B. and Seo, J. I. (2011a). Bayesian estimations on the exponentiated half triangle distribution under type-I hybrid censoring. Journal of Korean Data 83 Information Science Society, 22, 565-574.

Kim, Y., Kang, S. B. and Seo, J. I. (2011b). Bayesian estimation in the generalized half logistic distribution under progressively type-II censoring. Journal of Korean Data 8 Information Science Society, 22, 977-987.

Kus, C. and Kaya, M. F. (2006). Estimation of parameters of the loglogistic distribution based on progressive censoring using the EM algorithm. Hacettepe Journal of Mathematics and Statistics, 35, 203-211.

Mathai, A. M. (2003). Order statistics from a logistic distribution and applications to survival and reliability analysis. IEEE Transaction on Reliability, 52, 200-206.

Maswadah, M. (2003). Conditional confidence interval estimation for the inverse Weibull distribution based on censored generalized order statistics. Journal of Statistical Computation E Simulation, 12, 887-898. 
Nelson, W. (1982). Applied life data analysis, New York: Willy.

Pettitt, A. N. and Stephens, M. A. (1976). Modified Cramer-von Mises statistics for censored data. Biometrika, 63, 291-298.

Porter III, J. E., Coleman, J. W. and Moore, A. H. (1992). Modified KS, AD, and C-vM tests for the Pareto distribution with unknown location \& scale parameters. IEEE Transactions on Reliability, 41, 112-117.

Puig, P. and Stephens, M. A. (2000). Tests of fit for the Laplace distribution with applications. Technometrics, 42, 417-424.

Shin, H. and Lee, K. (2012). Estimation in the exponential distribution under progressive type I interval censoring with semi-missing data. Journal of Korean Data \& Information Science Society, 23, 12711277 . 